EPJ Web of Conferences 63, 01014 (2013)

DOI: 10.1051/epjconf/ 20136301014

(C) Owned by the authors, published by EDP Sciences, 2013

\title{
The prolate-oblate shape phase transition in the interacting boson model
}

\author{
Y. Zhang ${ }^{1, a}$, J. Xu' ${ }^{1}$, S. X. $\mathrm{Li}^{1}$, and Y. $\mathrm{An}^{1}$ \\ ${ }^{1}$ Department of Physics, Liaoning Normal University, Dalian 116029, China
}

\begin{abstract}
The prolate-oblate shape phase transition in the the interacting boson model is revisited by adopting the transitional Hamiltonian with a linear dependence on the control parameter. The analysis of the shape phase transition in both the large- $N$ limit and finite $N$ case shows that the $\mathrm{O}(6)$ symmetry is robust as the critical point symmetry of the prolate-oblate shape phase transition.
\end{abstract}

\section{Introduction}

Shape phase transitions in nuclei have attracted a lot of interest from both experimental and theoretical perspectives $[1,2,3]$. In theory, the most frequently used model for studying shape phase transition is the interacting boson model (IBM) [4], which possesses an overall U(6) symmetry with three dynamical symmetries (symmetry limits) corresponding three special nuclear shapes or collective modes; namely, a spherical vibrator [U(5)], a prolate rotor $[\mathrm{SU}(3)]$, and a $\gamma$-soft $[\mathrm{O}(6)]$. Thus, various nuclear shape phase transitions can be explored within the transitional patterns among different symmetries in the IBM. For example, the phase transition from spherical to axially deformed shape is characterized as the U(5)-SU(3) transition; the phase transition from spherical to the $\gamma$-soft motion is described by the $\mathrm{U}(5)-\mathrm{O}(6)$ transition; and the phase transition from prolate to oblate shape is often described by the $\mathrm{SU}(3)-\mathrm{O}(6)-\overline{\mathrm{SU}(3)}$ transition $[5,6,7,8]$, in which the prolate and oblate phase are described by the SU(3) and $\overline{\mathrm{SU}(3)}$ symmetry limit respectively, and the $\mathrm{O}(6)$ symmetry limit emerges exactly at the critical point since the traditional Hamiltonian is designed to pass the $\mathrm{O}(6)$ limit via a nonlinear dependence on the control parameter [5]. Particularly, the recent study [8] shows a new signature of the first order phase transition at the $\mathrm{O}(6)$ limit of the IBM. In addition, it should be emphasized that the $\overline{\mathrm{SU}(3)}$ dynamical symmetry can be realized from the traditional SU(3) dynamical group via a gauge transformation $[9,10,11,12]$.

In this work, we will investigate the prolate-oblate shape phase transition in the IBM by considering the Hamiltonian with a linear dependence on the control parameter to test the validity of the $\mathrm{O}(6)$ dynamics from a more general phase transitional perspective.

\footnotetext{
a e-mail: dlzhangyu_physics@163.com
}

\section{Model}

A Hamiltonian in the IBM framework is constructed from two kinds of boson operators; namely, a $s$-boson with $J^{\pi}=0^{+}$and a $d$-boson with $J^{\pi}=2^{+}$. The total boson number $N$ is taken as the number of valence particle (or hole) pairs in a nucleus. As mentioned above, the prolateoblate shape phase transition can be described with the $\mathrm{SU}(3)-\mathrm{O}(6)-\overline{\mathrm{SU}(3)}$ transition in the IBM. To realize such a transitional dynamics, Jolie et al suggest a simple Hamiltonian $[5,6,7]$ written as

$$
\hat{H}^{\chi}=-\varepsilon\left[\hat{Q}^{\chi} \cdot \hat{Q}^{\chi}\right],
$$

where

$$
\hat{Q}_{\mu}^{\chi}=\left(s^{\dagger} \times \tilde{d}+d^{\dagger} \times \tilde{s}\right)_{u}^{(2)}+\chi\left(d^{\dagger} \times \tilde{d}\right)_{u}^{(2)}
$$

is the quadrupole operator, $\varepsilon$ is a scale parameter, and $\chi$ is the control parameter. It can be proven $[5,7]$ that Eq. (1) is just the Hamiltonian in the SU(3) limit when $\chi=-\frac{\sqrt{7}}{2}$; it is in the $\overline{\mathrm{SU}(3)}$ limit when $\chi=\frac{\sqrt{7}}{2}$ and it is in the $\mathrm{O}(6)$ limit when $\chi=0$. For $\chi \in\left[-\frac{\sqrt{7}}{2}, \frac{\sqrt{7}}{2}\right]$, Eq. (1) can then be used to describe the $\mathrm{SU}(3)-\mathrm{O}(6)-\mathrm{SU}(3)$ transition, and the $\mathrm{O}(6)$ limit as the critical point symmetry of the phase transition is only a special case of Eq. (1) with $\chi=0$.

Generally, the form of the Hamiltonian used to describe the quantum phase transition from phase A to phase $\mathrm{B}$ is often built as [2]

$$
\hat{H}_{\mathrm{A}-\mathrm{B}}=\varepsilon\left[(1-x) \hat{H}_{\mathrm{A}}+x \hat{H}_{\mathrm{B}}\right],
$$

with $\hat{H}_{\mathrm{A}}$ and $\hat{H}_{\mathrm{B}}$ describing the phase $\mathrm{A}$ and $\mathrm{B}$ respectively. Clearly, it is a linear dependence on the control parameter in Eq. (3) in contrast to Eq. (1), in which the coupling between the two phases is in a nonlinear form via the control parameter $\chi$. In the many-body system, the linear ansatz is naturally satisfied if the control parameter represents a weight factor at a certain interaction term of the Hamiltonian $[2,13]$. In the following, we will revisit the prolateoblate shape phase transition in the IBM within a more 


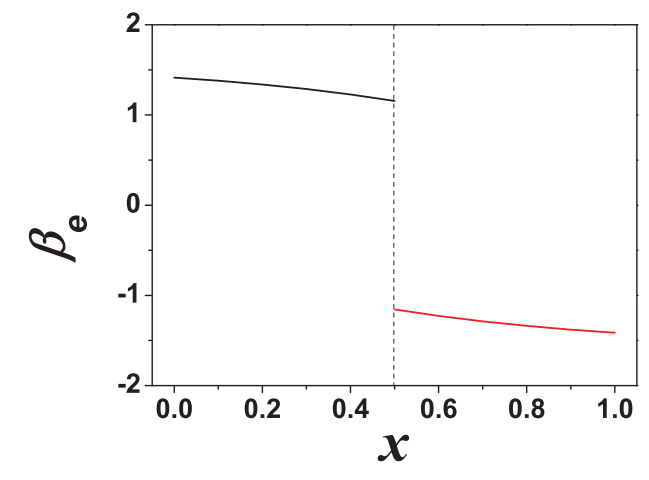

Figure 1. The classical order parameter $\beta_{e}$, which is defined as the optimal value of $\beta$, is shown as function of the control parameter $x$.
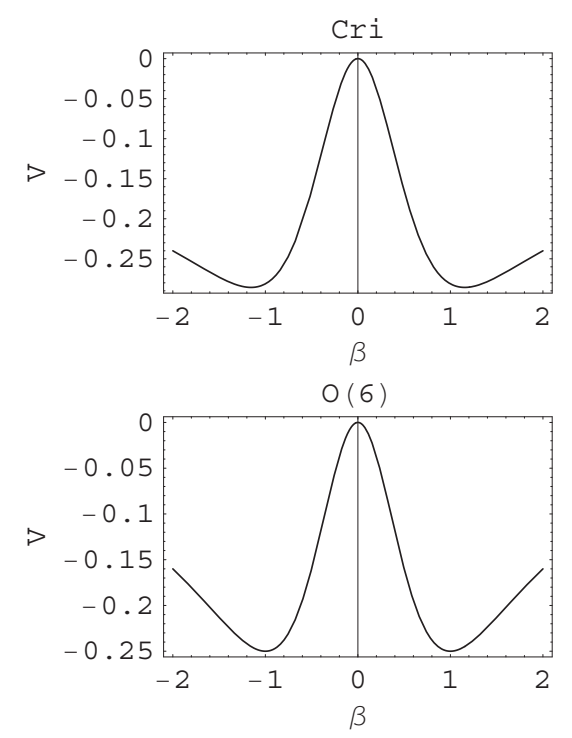

Figure 2. The potential function in $\beta$ obtained from (6) at the critical point $x=0.5$ and the potential in the $\mathrm{O}(6)$ limit corresponding to (1) with $\chi=0$.

generally parameterized pattern as given in Eq. (3). The Hamiltonian is constructed as

$$
\hat{H}_{x}=-\varepsilon\left[(1-x)\left[\hat{Q}^{\mathrm{SU}(3)} \cdot \hat{Q}^{\mathrm{SU}(3)}\right]+x\left[\hat{Q}^{\overline{\mathrm{SU}(3)}} \cdot \hat{Q}^{\overline{\mathrm{SU}(3)}}\right]\right],
$$

where $\hat{Q}^{\mathrm{SU}(3)}=\left(s^{\dagger} \times \tilde{d}+d^{\dagger} \times \tilde{s}_{u}^{(2)}-\frac{\sqrt{7}}{2}\left(d^{\dagger} \times \tilde{d}\right)_{u}^{(2)}\right.$ and $\hat{Q}^{\overline{\mathrm{SU}(3)}}=\left(s^{\dagger} \times \tilde{d}+d^{\dagger} \times \tilde{s}\right)_{u}^{(2)}+\frac{\sqrt{7}}{2}\left(d^{\dagger} \times \tilde{d}\right)_{u}^{(2)}$. It is clear that the case of $x=0$ corresponds the SU(3) limit (the prolate phase) and the case of $x=1$ represents the $\overline{\mathrm{SU}(3)}$ limit (the oblate phase). Then the shape phase transition from prolate to oblate may be described by Eq. (4) with $x \in[0,1]$. In this work, the scaled parameters are taken as $\varepsilon=\frac{1}{4 N}$ for all the Hamiltonian in concrete calculations.

\section{Shape phase transition and critical point dynamics}

To investigate the prolate-oblate shape phase transition in the large- $N$ limit, the coherent state (also called the intrinsic state) [4] defined as

$$
\begin{aligned}
|\beta, \gamma\rangle & =\frac{1}{\sqrt{N !\left(1+\beta^{2}\right)^{N}}} \\
& \times\left[s^{\dagger}+\beta \cos \gamma d_{0}^{\dagger}+\frac{1}{\sqrt{2}} \beta \sin \gamma\left(d_{2}^{\dagger}+d_{-2}^{\dagger}\right)\right]^{N}|0\rangle
\end{aligned}
$$

may be adopted to study the classical limit of the model. The scaled potential function corresponding to the Hamiltonian (4) in the large- $N$ limit is then estimated by the expectation value in the coherent state, $V(x, \beta, \gamma)=$ $\langle\beta, \gamma, N|\hat{H}| \beta, \gamma, N\rangle / N$. Thus, the potential in the large- $N$ limit can be expressed as

$$
\begin{aligned}
V(x, \beta, \gamma) & =-\frac{\beta^{2}}{8\left(1+\beta^{2}\right)^{2}}\left[\beta^{2}+4 \sqrt{2} \beta \cos 3 \gamma+8\right] \\
& +x \frac{\sqrt{2} \beta^{3} \cos 3 \gamma}{\left(1+\beta^{2}\right)^{2}} .
\end{aligned}
$$

To identify the order of phase transition, one should minimize the potential function (6) by varying $\beta$ and $\gamma$ for each $x$ value. The optimal values are denoted as $\beta_{e}$ and $\gamma_{e}$. Moreover, it is easy to prove that the simple form of the dependence on $\gamma$ in (6) yields either $\gamma_{e}=0$ for $x<0.5$ or $\gamma_{e}=\pi / 3$ for $x>0.5$. The later case can be equivalently described by substituting $\gamma_{e}=0$ and $\beta_{e}=-\beta_{e}$. As a result, one only needs to consider the potential evolution in $\beta$ to identify the order of the phase transition described by (6) after setting $\gamma=0$. Further, the optimal values $\beta_{e}$, which can be considered as the classical order parameter [14], are shown in Fig. 2 as function of $x$. One can clearly see from Fig. 2 that the prolate phase characterized with $\beta_{e}>0$ is always dominant for $x<0.5$, and the oblate phase represented by $\beta_{e}>0$ becomes dominant for $x>0.5$. Specifically, the order parameter $\beta_{e}$ may move discontinuously from a positive value to a negative value when crossing $x=0.5$, which indicates that it is a first order transition occurring at the critical point $x=0.5$ shown in Fig. 2 .

It is easy to know from (6) that the potential function at the critical point $x=0.5$ is $\gamma$-independent ( $\gamma$-soft). As we know, the potential in the $\mathrm{O}(6)$ limit is also $\gamma$-independent. Comparing the potentials in the two cases: the potential function at the critical point $x=0.5$ and the potential function in the $\mathrm{O}(6)$ limit are shown in Fig. 2. As seen from Fig. 2, the global features of the potential at the critical point are almost the same as those of the $\mathrm{O}(6)$ potential except for that the double wells in the former seem to be shallower than those in the latter.

Analysis indicates that a first-order shape phase transition dose occurs in the boson system described by (4) in the large- $N$ limit with the potential at the critical point very similar to that in the $\mathrm{O}(6)$ limit. However, the boson number $N$ is always finite in realistic systems. To study the shape phase transition and the associated critical dynamics in finite $N$ case, one needs to numerically solve the Hamiltonian. In finite $N$ case, the ground state energy 

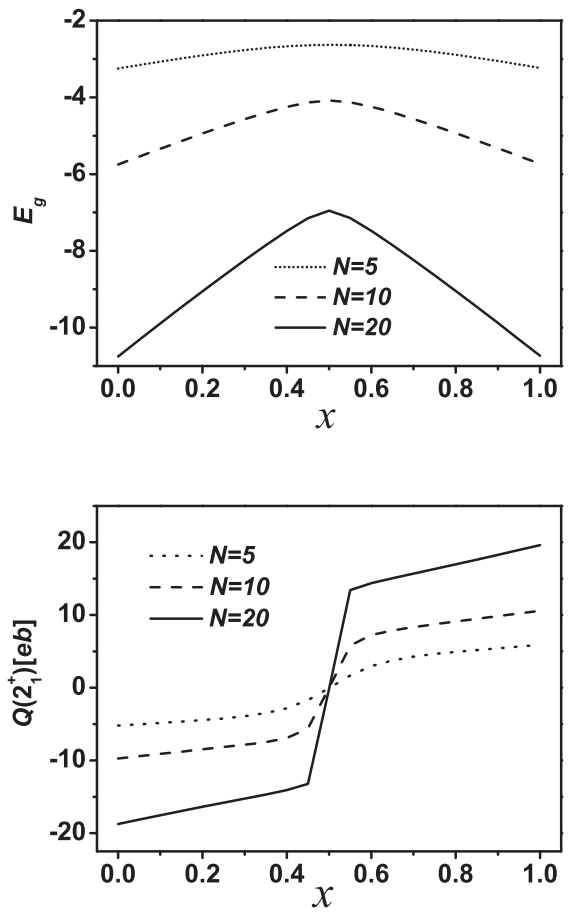

Figure 3. The ground state energy $E_{q}$ and qudropole moment $Q\left(2_{1}^{+}\right)$are shown as function of $x$ for $N=5,10,20$, where the effective charge is taken as $t=1 \mathrm{eb}$.

may play the same role as done by the potential function in the classical limit, of which the derivatives vs $x$ determines the order of phase transition. On the other hand, the quadrupole moment $Q\left(2_{1}^{+}\right)$of the first $2^{+}$state can be used to measure the prolate-oblate shape phase transition in experiments. $Q\left(2_{1}^{+}\right)<0$ indicates a prolate phase and $Q\left(2_{1}^{+}\right)>0$ represents an oblate phase. Thus, we calculate the two quantities and show them in Fig. 3 as functions of $x$ for boson number $N=5,10,20$. For the electromagnetic observables, the electric quadrupole transitional operator is chosen as

$$
T(E 2)=t\left[(1-x) \hat{Q}^{\mathrm{SU}(3)}+x \hat{Q}^{\overline{\mathrm{SU}(3)}}\right],
$$

which is consistent with the form of the Hamiltonian (4). As seen in Fig. 3, $E_{g}$ presents a non-monotonic variation as function of $x$. Specifically, at $x=0.5$, the derivative $\frac{\partial E_{g}}{\partial x}$ may change its sign and tend to be discontinue in the large $N$ case such $N=20$. One can also find from Fig. 3 that there is a sudden change in the values of the quadropole moment $Q\left(2_{1}^{+}\right)$around $x_{c}=0.5$ from the negative to the positive. The above results indicate the additional evidences of the prolate-oblate phase transitional behavior in finite systems [8].

Further derivation shows that the Hamiltonian (4) at the critical point $x=0.5$ can be expressed as

$$
H_{\mathrm{cri}}=H_{\mathrm{O}(6)}-\varepsilon \frac{7}{4}\left(d^{\dagger} \times \tilde{d}\right)^{(2)} \cdot\left(d^{\dagger} \times \tilde{d}\right)^{(2)},
$$

Table 1. The energy ratios $R_{4 / 2}=E_{4_{1}} / E_{2_{1}}, R_{6 / 2}=E_{61} / E_{2_{1}}$ and $R_{6 / 0}=E_{61} / E_{0_{2}}$ as well as the typical $B(E 2)$ ratios are shown for $N=20$, where $A_{\text {cri }}$ and $A_{\mathrm{O}(6)}$ represent the quantities calculated from $H_{\text {cri }}$ and $H_{\mathrm{O}(6)}$ respectively.

\begin{tabular}{lccccc}
\hline & $R_{4 / 2}$ & $R_{6 / 2}$ & $R_{6 / 0}$ & $\frac{B\left(E 2 ; 4_{1} \rightarrow 2_{1}\right)}{B\left(E 2 ; 2_{1} \rightarrow 0_{1}\right)}$ & $\frac{B\left(E 2 ; 0_{2} \rightarrow 2_{1}\right)}{B\left(E 2 ; 2_{1} \rightarrow 0_{1}\right)}$ \\
\hline$A_{\text {cri }}$ & 2.35 & 4.04 & 0.76 & 1.43 & 0.00 \\
$A_{\mathrm{O}(6)}$ & 2.50 & 4.50 & 1.00 & 1.41 & 0.00 \\
\hline
\end{tabular}

where

$$
H_{\mathrm{O}(6)}=-\varepsilon \hat{Q}^{0} \cdot \hat{Q}^{0}
$$

is the Hamiltonian in the $\mathrm{O}(6)$ limit obtained from (1) with $\chi=0$ [5]. In (9), $\hat{Q}_{u}^{0}=\left(s^{\dagger} \times \tilde{d}+d^{\dagger} \times \tilde{s}\right)_{u}^{(2)}$ is the generator of $\mathrm{O}(6)$ algebra. It is clear that the second term in (8) makes the dynamics at the critical point different from the one in the $\mathrm{O}(6)$ limit. To identify the effects of this term on the $\mathrm{O}(6)$ dynamics, we list in Table 1 some typical energy ratios and $B(E 2)$ ratios calculated by $H_{\text {cri }}$ and $H_{\mathrm{O}(6)}$ respectively. As seen in Table 1, the values of $E_{4_{1}} / E_{2_{1}}, E_{6_{1}} / E_{2_{1}}$ and $E_{6_{1}} / E_{0_{2}}$ for the critical point are all lower than those in the $\mathrm{O}(6)$ limit. Especially, the degeneration of energy levels characterized with $R_{6 / 0}=1.00$ in the $\mathrm{O}(6)$ limit is evidently broken at the critical point. However, the average deviation between the two cases defined as $\frac{1}{n} \sum_{i}\left|\frac{A_{\mathrm{O}(6)}-A_{\text {cri }}}{A_{\mathrm{O}(6)}}\right|$ is less than $14 \%$ for the three energy ratios. In addition, the values of $B(E 2)$ ratios at the critical point are almost the same as those in the $\mathrm{O}(6)$ limit, which indicates that components of the wave-functions in the two cases are very similar at least for the low-lying states.

\section{Conclusion}

In conclusion, we have analyzed the prolate-oblate shape phase transition within the framework of the IBM by using the Hamiltonian with a linear dependence on the control parameter in contrast to the original $\mathrm{SU}(3)-\mathrm{O}(6)-\overline{\mathrm{SU}(3)}$ transitional pattern $[5,6,7,8]$, in which the adopted Hamiltonian is designed with a nonlinear dependence on the control parameter. The results in the large $N$ limit indicate that the prolate-oblate phase transition shown in this pattern is of first order similar to that shown in the traditional scheme $[5,6,7,8]$, and the resulting $\gamma$-soft critical dynamical structure in the finite cases is also similar to that of the $\mathrm{O}(6)$ limit.

Support from the Natural Science Foundation of China (11005056,11375005) is acknowledged

\section{References}

[1] R. F. Casten and E. A. McCutchan, J. Phys. G 34, R285 (2007).

[2] P. Cejnar and J. Jolie, Prog. Part. Nucl. Phys. 62, 210 (2009).

[3] P. Cejnar, J. Jolie and R. F. Casten, Rev. Mod. Phys. 82, 2155 (2010).

[4] F. Iachello and A. Arima, The Interacting Boson Model (Cambridge University, Cambridge, England, 1987). 
[5] J. Jolie, R. F. Casten, P. von Brentano, and V. Werner, Phys. Rev. Lett. 87, 162501 (2001).

[6] J. Jolie and A. Linnemann, Phys. Rev. C 68, 031301(R) (2003).

[7] F. Pan, T. Wang, Y. S. Huo, and J. P. Draayer, J. Phys. G 35, 125105 (2008).

[8] L. Bettermann, V. Werner, E. Williams, and R. J. Casperson, Phys. Rev. C 81, 034326(R) (2010).

[9] D. Kusnezov, Phys. Rev. Lett. 79, 537 (1997).
[10] A. M. Shirokov, N. A. Smirnova, and Y. F. Smirnov, Phys. Lett. B 434, 237 (1998).

[11] P. Van Isacker, A. Frank, and J. Dukelsky, Phys. Rev. C 31, 671 (1985).

[12] P. Cejnar and J. Jolie, Phys. Lett. B 420, 241 (1998).

[13] P. Cejnar, S. Heinze, and J. Jolie, Phys. Rev. C 68, 034326 (2003).

[14] F. Iachello and N. V. Zamfir, Phys. Rev. Lett. 92, 212501 (2004). 